\title{
Task-related modulation of anterior theta and posterior alpha EEG reflects top-down preparation
}

\author{
Byoung-Kyong Min ${ }^{1}$ and Hae-Jeong Park*2
}

\begin{abstract}
Background: Prestimulus EEG alpha activity in humans has been considered to reflect ongoing top-down preparation for the performance of subsequent tasks. Since theta oscillations may be related to poststimulus top-down processing, we investigated whether prestimulus EEG theta activity also reflects top-down cognitive preparation for a stimulus.

Results: We recorded EEG data from 15 healthy controls performing a color and shape discrimination task, and used the wavelet transformation to investigate the time course and power of oscillatory activity in the signals. We observed a relationship between both anterior theta and posterior alpha power in the prestimulus period and the type of subsequent task.

Conclusions: Since task-differences were reflected in both theta and alpha activities prior to stimulus onset, both prestimulus theta (particularly around the anterior region) and prestimulus alpha (particularly around the posterior region) activities may reflect prestimulus top-down preparation for the performance of subsequent tasks.
\end{abstract}

\section{Background}

When people identify an object, they have to match what they sense against their knowledge. In general, people accomplish recognition by a combination of two information processing pathways: top-down and bottom-up. Bottom-up processing (i.e. sensation) occurs when the sensory input information induces perceptual representation, whereas top-down processing (i.e. identification) occurs when the perceptual representation is influenced by some higher mental function such as previous knowledge, motivation, or expectation.

To facilitate perceptual identification, one may use subjective expectations of the stimulus to come. Accordingly, a cognitive intention (e.g. expectation, mental readiness, active redirection of attention), embedded in a top-down process, may precede an event or stimulus. Bottom-up sensory processing is then guided by such top-down processing as a specific reallocation of attention relevant to the type of stimulus to follow or task to be performed. In this way, top-down intentional processing can increase the speed and efficiency of perceptual identification.

\footnotetext{
*Correspondence: parkhj@yuhs.ac

2 Brain Korea 21 Project for Medical Science, Department of Radiology, Nuclear Medicine and Research Institute of Radiological Science, Yonsei University College of Medicine, Seoul, Korea

Full list of author information is available at the end of the article
}

In this regard, the influence of prestimulus mental activity on the subsequent poststimulus responses (or task-performances) is worth investigating. Indeed, some studies showed the relationship between the prestimulus alpha activity and the poststimulus event-related potential (ERP) components. Brandt's and Barry's groups have reported that greater prestimulus alpha amplitude led to larger ERP amplitudes [1-5], whereas Başar's group has found an inverse relation between prestimulus rootmean-square (RMS) alpha power and subsequent ERP amplitudes [6-9]. As it is known, ERPs are based on the recording of brain electrical potentials synchronized with the presentation of external sensory stimuli (so-called 'exogenous') as well as the occurrence of internal cognitive events (so-called 'endogenous') [10-12]. Likewise, there is still a fundamental debate on whether ERP components are generated from the ongoing EEG activity (e.g. alpha) by means of phase-reset [13-19]. In general, ERP components are influenced by bottom-up (sensory or physical) factors and also reflect top-down (cognitive) processing. However, since those studies show inconsistent observations, the relationship between the prestimulus alpha power and the poststimulus ERP components has been still controversial.

Meanwhile, using a color-shape discrimination task, Min and Herrmann [20] recently found that the prestim- 
ulus level of alpha activity differed significantly with the type of poststimulus task, and interpreted this to mean that prestimulus alpha activity reflects top-down preparation for the stimulus. Accordingly, the prestimulus mental state, typically regarded as a resting state and thus a baseline for poststimulus activity, may in fact be a taskrelevant top-down control stage. Some previous studies consistently support this conception [21,22]. For instance, Ergenoglu et al. [21] observed that alpha activity modulates visual detection performance in humans.

Besides, Klimesch et al. [23] postulated that alpha synchronization might reflect a top-down function in inhibiting task-irrelevant information, since the event-related synchronization in the alpha band can be noticeably observed during task-performance either under such conditions where subjects have to withhold task-relevant information or over the brain regions that are task-irrelevant [24-30]. Therefore, by means of two kinds of discrimination tasks requiring inhibition of concurrent taskirrelevant feature processing for improving task-performance, here we would like to test a putative relationship between prestimulus EEG dynamics and poststimulus responses of task-performance from the viewpoint of top-down inhibitory preparation to the task-irrelevant feature of subsequently presented stimuli. Moreover, Mordkoff and Yantis [31] reported that coactivation occurs when target attributes from two separable dimensions are simultaneously present, but not when target attributes come from the same dimension. They argued dividing attention between color and shape as evidence of coactivation. To induce such coactivation, we employed target attributes from two dimensions (color and shape). This required subjects to inhibit the task-irrelevant feature to improve performance. Presumably, different taskdifficulties across the color and shape tasks may induce differences in top-down preparation such as prestimulus task-performance strategy.

In addition, theta activity has received attention as a possible electrophysiological correlate of top-down processing. It has been reported that spontaneous EEG theta activity influences the amplitude of frontal visual evoked potentials [32], and that Freunberger et al. [33] suggested that phase-locked theta activity reflects top-down regulation in information memory systems. Sauseng et al. [34] also suggested that theta activity may represent top-down processing, and gamma activity bottom-up processing, based on observations of enhanced phase-synchrony between theta and gamma bands for an attended visual target. Studies consistently suggest that slow oscillations (e.g. theta and alpha) are related to function in large, distributed networks [35,36], whereas high frequency oscillations (e.g. gamma) are associated with neural processes in more local networks [36]. These studies support the conclusion that integration between top-down processes depends critically on slow oscillations such as alpha and theta.

Most studies on theta oscillations as a measure of topdown processes have focused on event-related (or poststimulus) modulations, and thus neglect prestimulus theta activity as ongoing top-down preparation for the performance of subsequent tasks. Note that a prestimulus mental state differs from a spontaneous mental state (i.e. a resting state with no tasks) in that mental activity in a prestimulus period would be influenced by the attributes of subsequent tasks or events. The prestimulus mental state may therefore present an appropriate target to explore differences in top-down regulation in advance of different task performances. We hypothesized that if theta activity reflects top-down processing, it must be largely controlled by ongoing top-down inhibitory processing, particularly during a prestimulus mental state almost entirely lacking in physical attributes of upcoming stimuli (i.e. bottom-up processes). In the present study we therefore investigated whether prestimulus theta activity represents a state of cognitive readiness, as prestimulus alpha activity does [20].

\section{Results}

Pairs of colored figures randomly drawn from a set of red or green circles or squares were presented, and participants were requested to respond with the index finger of one hand if the target feature of the task ('color' in the color task and 'shape' in the shape task) was the same and to respond with the other hand if it was not. As a result, the shape task yielded significantly longer reaction times than the color task $(\mathrm{F}(1,14)=5.2, p<0.05$; color task: 486 ms, shape task: $528 \mathrm{~ms})$. For the accuracy of the task-performance, we also found a strong 'task' effect $(\mathrm{F}(1,14)=$ 15.0, $p<0.005)$, indicating that the color task performance showed significantly higher accuracy than the shape task performance (color task: $96.6 \%$, shape task: $74.2 \%)$.

As shown in Figs. 1 and 2, we observed the significant main effect of 'task' $(\mathrm{F}(1,14)=5.1, p<0.05)$ with higher prestimulus total alpha power for the shape task (color task: $10.9 \mu \mathrm{V}^{2}$, shape task: $18.7 \mu \mathrm{V}^{2}$ ). We also found that the shape task was preceded by significantly higher prestimulus total theta power than the color task $(\mathrm{F}(1,14)=$ 5.0, $p<0.05$; color task: $6.0 \mu \mathrm{V}^{2}$, shape task: $7.7 \mu \mathrm{V}^{2}$ ). In addition, we observed that the anterior ROI showed significantly higher prestimulus total theta power than the posterior ROI $(\mathrm{F}(1,14)=10.0, p<0.01$; anterior ROI: 10.2 $\mu \mathrm{V}^{2}$, posterior ROI: $3.5 \mu \mathrm{V}^{2}$; cf. Figs. 1 and 2 ).

Compared to the posterior region, the anterior region showed significantly higher poststimulus total power of both alpha $\left(\mathrm{F}(1,14)=4.8, p<0.05\right.$; anterior ROI: $12.9 \mu \mathrm{V}^{2}$, posterior ROI: $\left.10.4 \mu \mathrm{V}^{2}\right)$ and theta activity $(\mathrm{F}(1,14)=5,0$, $p<0.05$; anterior ROI: $19.1 \mu \mathrm{V}^{2}$, posterior ROI: $14.6 \mu \mathrm{V}^{2}$ ). 


\section{(A) Prestimulus theta power}

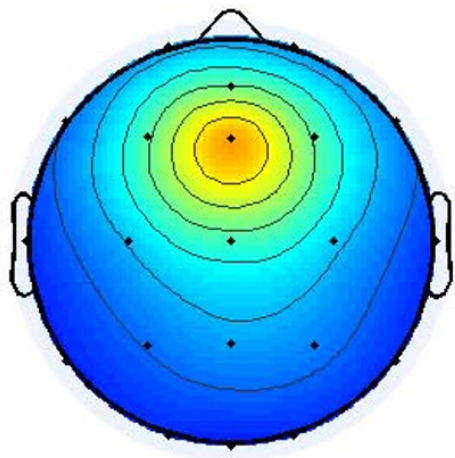

Color task

Color task

\section{(B) Prestimulus alpha power}

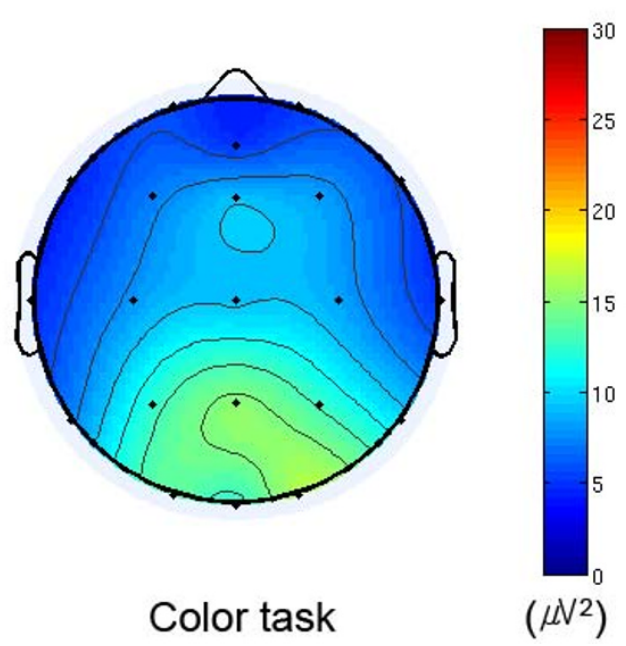

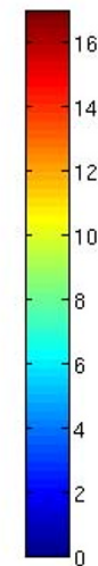

$\left(\mu \mathrm{N}^{2}\right)$

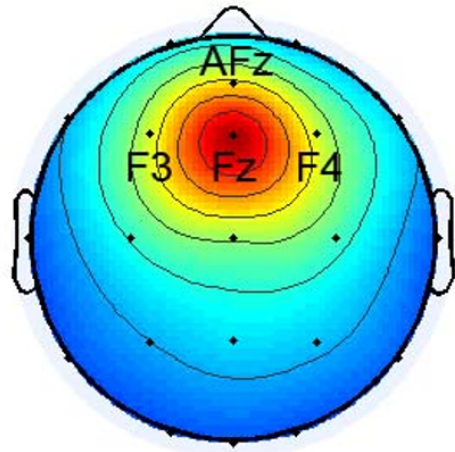

Shape task

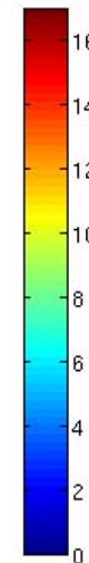

$\left(\mu \mathrm{N}^{2}\right)$
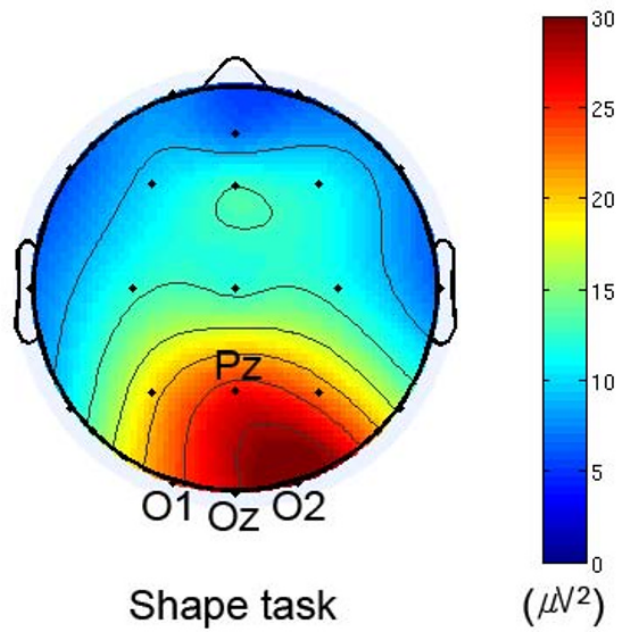

$\left(\mu \mathrm{V}^{2}\right)$

Figure $1 \mathrm{Grand}$-averaged topographies of (A) prestimulus theta and (B) prestimulus alpha power for both tasks. These topographical distributions were computed by averaging the mean power at subjects' individual alpha and theta frequencies over the time window from 300 to $50 \mathrm{~ms}$ prestimulus. Within this time window, individual alpha and theta frequencies were obtained from the frequencies showing maximal power of each task in alpha band on the electrode $\mathrm{Oz}$ and in theta band on the electrode $\mathrm{Fz}$, respectively. Note the differences in prestimulus alpha power around the posterior region (including the electrodes $\mathrm{Pz}, \mathrm{O} 1, \mathrm{Oz}$ and $\mathrm{O} 2$ ), and those of prestimulus theta power around the anterior region (including the electrodes AFz, F3, Fz and F4) between the two tasks. All views are from the vertex, and the upside is nasal. Color bars indicate scales of the power $\left(\mu \mathrm{V}^{2}\right)$ of oscillatory activity.

In addition, the shape task showed significantly lower poststimulus alpha power than the color task $(\mathrm{F}(1,14)=$ 7.9, $p<0.05$; color task: $12.3 \mu \mathrm{V}^{2}$, shape task: $\left.11.0 \mu \mathrm{V}^{2}\right)$. However, the 'task' factor did not have a significant effect on the poststimulus theta power.

The topographical distributions for both alpha and theta power between the two tasks are presented in Fig. 1. As shown, the prestimulus anterior theta and posterior alpha power were more intense in the shape task than in the color task. Fig. 2 shows grand-averaged time-fre- quency representations of power for total activity on the anterior and posterior ROIs. Note that the theta power had its maximum after the stimulation, whereas the alpha power showed dominance and higher power before stimulation.

\section{Discussion}

We observed more intense anterior theta and posterior alpha power in the prestimulus phase of the shape task than of the color task. One might possibly suspect that 


\section{(A) Anterior ROI}

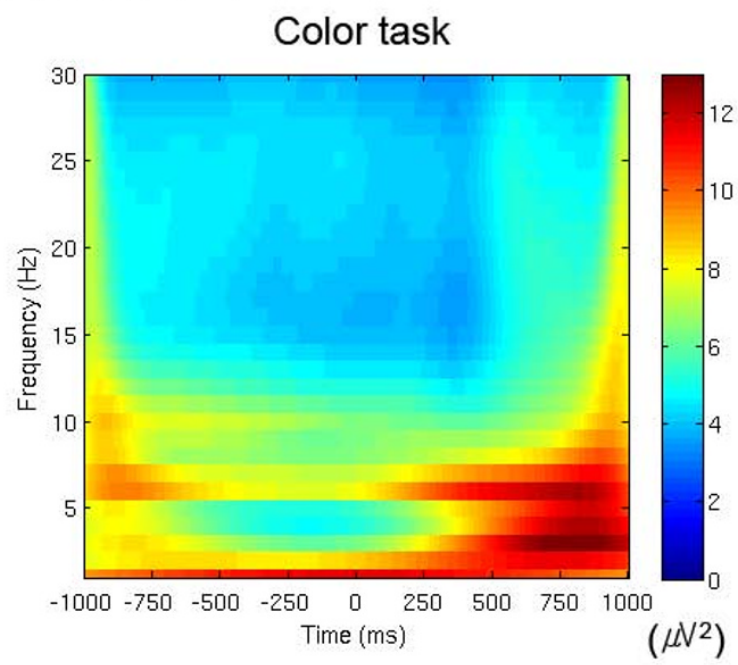

(B) Posterior ROI

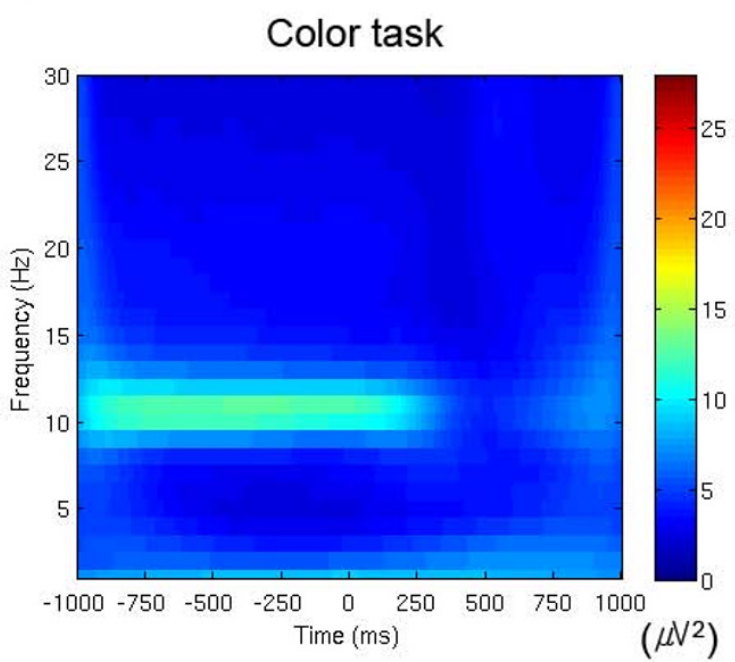

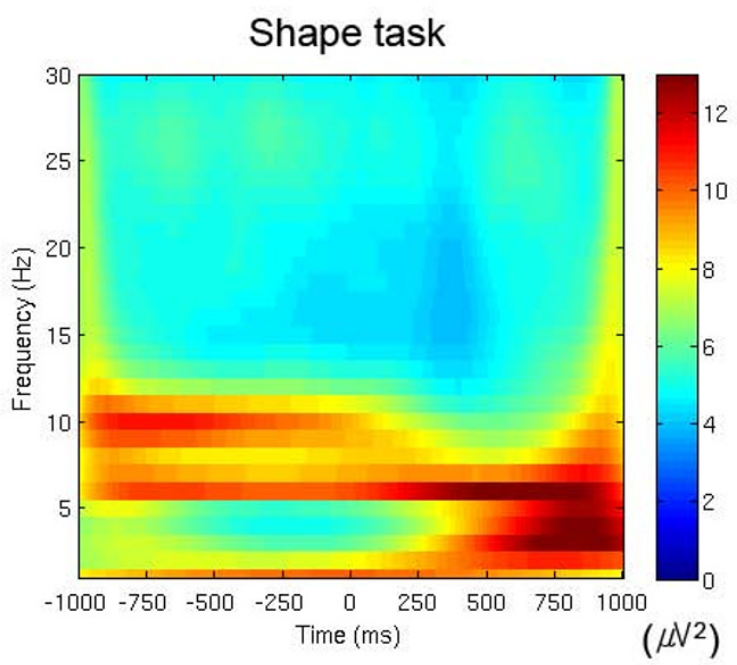

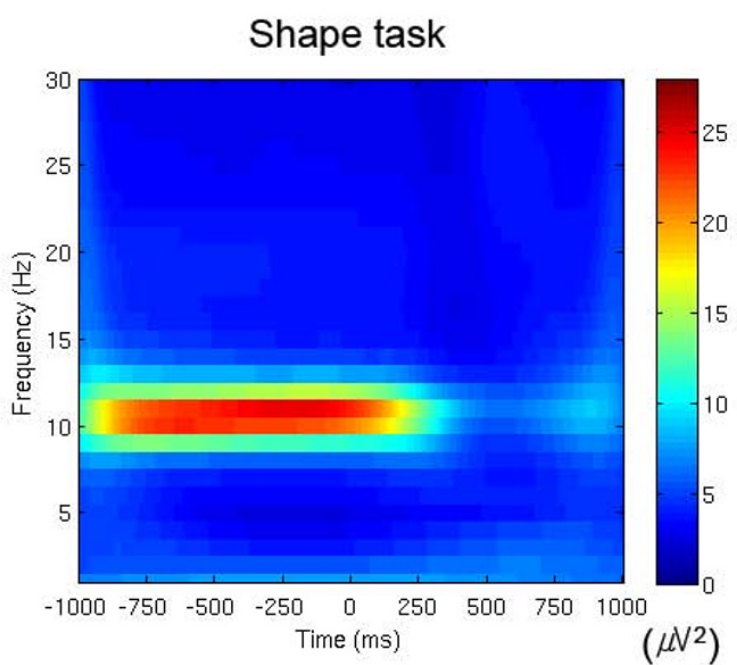

Figure 2 Grand-averaged time-frequency representations of total power of oscillatory activity $(1-30 \mathrm{~Hz})$ on (A) the anterior ROI and (B) the posterior ROI for both tasks. Stimuli were presented from 0 to $500 \mathrm{~ms}$. Color bars indicate scales of the power ( $\mu \mathrm{V} 2)$. Note the alpha activity (about $10 \mathrm{~Hz}$ ) on the posterior $\mathrm{ROI}$ (averaged across the electrodes $\mathrm{Pz}, \mathrm{O} 1, \mathrm{Oz}$ and $\mathrm{O} 2$ ) and the theta activity (about $6 \mathrm{~Hz}$ ) on the anterior ROI (averaged across the electrodes AFz, F3, Fz and F4), and compare the differences between the two tasks, particularly before stimulus onset.

the effects of prestimulus activity come from prolonged poststimulus responses. To validate the present effects as prestimulus effects, we performed an additional experiment with longer inter-stimulus intervals (ISIs; 2500$3500 \mathrm{~ms}$ as compared with the present ISIs of 1500-2500 $\mathrm{ms})$. Eventually, the same results were consistently obtained although we replicated the experiment with longer ISIs (Min et al., unpublished observations). Therefore, one can exclude a possibility that prestimulus effects are confounded by tailing of poststimulus responses. Moreover, since the prestimulus oscillatory activity was mea- sured sufficiently far from the mean latency for buttonresponses (about $507 \mathrm{~ms}$ poststimulus; subsequently the response-prestimulus time interval ranged approximately from 2200 to $3200 \mathrm{~ms}$ ), our observations in the prestimulus period are also out of the possible confounding ranges by response-related brain activity.

Based on behavioral results, the color feature may be more salient, and thus harder to ignore than the shape feature, in the successful performance of the shape task [20]. To suppress such salient task-irrelevant information as color, the shape task may demand more attention- 
readiness. The present study extends previous findings that prestimulus alpha activity reflects 'cognitive readiness' for an upcoming task [20,37-40], with evidence that prestimulus theta activity may have similar functional significance. It is noteworthy that theta power showed its greatest intensity in the medial frontal region (see topographies, cf. Fig. 1A). Based on previous studies [41,42], frontal midline theta activity seems to principally reflect alternative activation of the anterior cingulate cortex (ACC) and prefrontal cortex. Wang et al. [43] suggested that theta activity from the ACC might reflect active inhibition. They observed transient phase locking of task-relevant theta activity in relation to active inhibition in superficial cingulated layers. Yordanova et al. [44] also proposed that theta oscillations particularly in relation to movement execution are functionally associated with error monitoring, of which the ACC is mainly in charge [45]. In line with these findings, Hanslmayr et al. [46] reported that theta activity in the ACC increased linearly with increasing interference during the stroop task, and concluded that sustained phase coupling between the ACC and the prefrontal cortex most likely reflects the engagement of cognitive control mechanisms. These findings support a significant relationship between frontal midline theta activity and inhibitory control of taskirrelevant processing. As task difficulty increased in the present study, the demand for inhibitory control in cognitive preparation most likely increased, and resulted in significant differences in theta power levels between the two tasks. This interpretation is in accordance with the assumption by Klimesch et al. [26] that theta power reflects (at least in part) task difficulty and cognitive load.

A recent EEG-fMRI study [47] also showed enhancement of frontal theta and occipital alpha power during a modified Sternberg working memory task. While we observed modulations of anterior theta and posterior alpha power in the prestimulus period, they reported similar phenomena in the 'maintenance' phase during a working memory task. Our present observations in the prestimulus period and their observations in a working memory retention phase are all consistent with modulation of alpha and theta frequency bands depending on the current cognitive load. Understandably, the mental load increases with the attention a task demands, and induces greater alertness, which can help either strengthened inhibition of task-irrelevant processing or enhanced attention to task-relevant processing. These different experimental phases (i.e. 'prestimulus'; versus 'retention' period) may in fact represent analogous mental states, and thus share in part the same neural networks.

To integrate top-down influences into bottom-up information requires long-range communications, which slow oscillations such as alpha and theta activities might achieve $[35,36,48]$. The interplay between alpha and theta oscillations may, for example, reflect the transfer of information between memory systems [49], and the central executive functions of working memory may be reflected in the fronto-parietal coherence in alpha and theta bands $[35,50]$. Since the functional coupling between anterior and posterior brain regions may be essential to accomplish top-down control [51], the interactions between anterior theta and posterior alpha activities and topdown regulation processing must be explored in future research.

\section{Conclusions}

The present study extends our previous findings that prestimulus alpha activity reflects ongoing top-down preparation for the performance of upcoming tasks [20,40], by evidence that prestimulus theta activity may have similar functional significance. Based on both behavioral and electrophysiological results, the difficult task (shape task), which requires more inhibition of the competing color feature, was preceded by significantly higher alpha and theta power as compared to the easy task (color task). Since such task-differences were reflected in both alpha and theta activities prior to stimulus onset, both prestimulus alpha and prestimulus theta activities may reflect top-down cognitive processing in preparation for the performance of subsequent tasks.

\section{Methods}

\section{Subjects}

Fifteen normal healthy volunteers ( 9 females, mean age 23; range 18-29 years) participated in this study, in accordance with the ethics guidelines at the Institutional Review Board of Yonsei University and the Declaration of Helsinki (World Medical Association: Ethical Principles for Medical Research Involving Human Subjects, 1964). Subjects gave informed consent prior to the start of the experiment. All had normal or corrected-to-normal vision, and none was color-blind, as determined by the Ishihara color test. None had a personal or family history of psychiatric disorders.

\section{Stimuli and procedure}

This study employed the same experimental paradigm as Min et al. [20,40] did. Pairs of colored figures randomly drawn from a set of red or green circles or squares were used as stimuli (cf. Fig. 3). The areas of circles and squares were matched. Stimuli were presented for 500 $\mathrm{ms}$, on a computer monitor placed in front of the subject at a distance of one meter. Two colored figures (a stimulus set) were presented side-by-side on a light-gray background at an eccentricity of $3^{\circ}$ (visual angle), and each colored figure in a stimulus set subtended a $4^{\circ}$ visual angle. All types of stimuli appeared approximately at random, with equal probability. Each stimulus presentation 


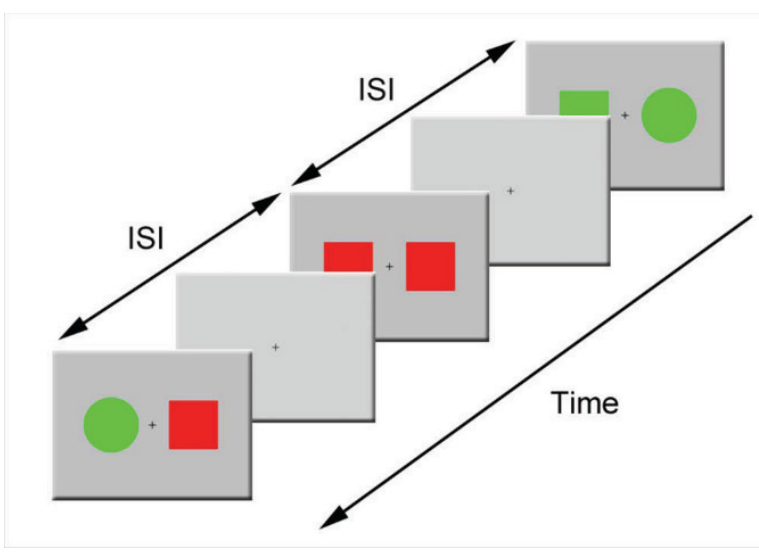

Figure $3 \mathrm{~A}$ task flow diagram of sample stimuli and the ISIs. TwO stimuli randomly drawn from a set of red or green circles or squares were presented bilaterally against a light-gray background on a computer monitor. Stimulus presentation was followed by a fixation cross presented during every ISI. The ISIs ranged randomly from 1500 to $2500 \mathrm{~ms}$.

was followed by variable ISIs ranging randomly between 1500 and $2500 \mathrm{~ms}$. We instructed subjects to remain centrally fixated and to press a button with the index finger of one hand if the target feature of the two presented stimuli ('color' in the color task and 'shape' in the shape task) was the same across the two presented stimuli, and to press another button with the other hand if it was not. Subjects were asked to press the button as quickly as possible. To limit the experimental paradigm to top-down processing, we employed the same physical stimuli across both tasks while the subjects performed different tasks.

The experiment consisted of two task-sessions: a color task and a shape task. Stimuli in each task were presented in two blocks separated by short rest periods. Response hands and the order of tasks were counterbalanced across subjects. The experiment consisted of 384 trials for each task. Only trials with correct responses were further analyzed.

\section{EEG recording}

EEG was recorded using a GRASS 15A54 amplifier (Grass Technologies, USA) with 21 sintered Au/Ag-electrodes. Their locations, in accordance with the international 10-20 system were as follows: AFz, Fp1, Fp2, Fz, F3, F4, F7, F8, Cz, C3, C4, T3, T4, Pz, P3, P4, T5, T6, Oz, O1 and $\mathrm{O} 2$. We also placed an electrode on each mastoid for the linked reference and a ground electrode at nasion. Eye movement activity was monitored with two additional electrodes placed supra-orbitally on both eyes and referenced to the linked mastoids. Electrode impedances were kept below $10 \mathrm{k} \Omega$ prior to data acquisition. EEG was sampled at $1000 \mathrm{~Hz}$ (analogue band-pass filter 0.1-100 Hz) and stored for off-line analysis. Data were epoched from $1000 \mathrm{~ms}$ prestimulus to $1000 \mathrm{~ms}$ poststimulus. Epochs containing eye-movements or other artifacts (maximum amplitude $\pm 70 \mu \mathrm{V}$ or electrode drifts) were rejected (As a result, the average rejection rate was $25.6 \%$ ).

\section{Wavelet transformation}

The power of oscillatory activity was investigated by convolving the EEG signals with Morlet wavelets $[52,53]$. The Morlet-convolved signal shows a Gaussian envelope with a temporal standard deviation $\left(\sigma_{t}\right)$ and a spectral standard deviation $\left(\sigma_{\mathrm{f}}=1 /\left(2 \pi \sigma_{\mathrm{t}}\right)\right)$ around its central frequency $\left(f_{0}\right)$ :

$$
\Psi(t, f)=A \exp (i 2 \pi f t) \exp \left(-t^{2} / 2 \sigma_{t}^{2}\right) .
$$

To have unit energy at all scales, the wavelet functions should be normalized prior to the convolution. For the Morlet wavelet transform, the normalization parameter $A$ is $\sigma_{\mathrm{t}}^{-1 / 2} \pi^{-1 / 4}$. A wavelet family is characterized by a constant ratio $\left(f_{0} / \sigma_{\mathrm{f}}\right)$, and we employed a wavelet family with $f$ ${ }_{0}$ ranging from 1 to $30 \mathrm{~Hz}$ in $1 \mathrm{~Hz}$ steps (cf. Fig. 2) and 3.8 as its constant ratio in order to consider optimal spectraltemporal resolution of lower frequency bands such as theta and alpha activities [54].

The wavelet transform was performed for each individual trial, and the absolute values of the resulting transforms were averaged. This measure of signal amplitude in single trials reflects the total activity for a certain frequency range, irrespective of whether it is phase-locked to the stimulus or not. We will refer to this measure as the total activity, since it includes evoked as well as induced activity. In the present study, we computed the power $\left(\mu \mathrm{V}^{2}\right)$ of oscillatory activity.

\section{Analytic methods}

Since we were interested in EEG oscillatory activity around both anterior and posterior regions, we selected for further analyses the two regions of interest (ROIs): anterior ROI (including the electrodes $\mathrm{AFz}, \mathrm{F} 3, \mathrm{Fz}$ and F4) and posterior ROI (including the electrodes $\mathrm{Pz}, \mathrm{O} 1$, $\mathrm{Oz}$ and $\mathrm{O} 2$ ), which showed most pronounced responsiveness to prestimulus theta and alpha power, respectively (cf. Fig. 1). In the present study, we confined alpha activity to the frequency range from 8 to $13 \mathrm{~Hz}$ and theta activity to the frequency range from 4 to $7 \mathrm{~Hz}$. For the prestimulus total power of these activities, we computed mean power in the time window from 300 to $50 \mathrm{~ms}$ prior to stimulus onset in each frequency range. This time window was chosen to avoid the temporal smearing of poststimulus activity into the baseline [52], while trying to take the time window as close to the stimulus onset as possible and also to include a reasonable period that included at least one cycle of both alpha and theta frequencies. No baseline correction was applied to the total power, since total alpha power in a prestimulus period 
would vanish after baseline correction. For the poststimulus total theta power, we measured maximum theta power in the time window from 200 to 450 ms poststimulus. This time window was determined on the basis of the grand averages (most pronounced and free of offset responses) and the previous studies about task-related (late) theta response were taken into account $[54,55]$. In the case of the poststimulus total alpha power, we chose the same time window, when the poststimulus amplitude modulation of alpha activity was most pronounced, and took minimum alpha power, since the grand-average of total alpha activity decreased after stimulation, as shown in Figs. 1 and 2. This phenomenon is typically referred to as 'alpha-blocking'.

Reaction times and accuracy of task-performance (error rates) were also measured for the behavioral analysis. Reaction times were collected within their individual 95\% confidence intervals. These behavioral measures were analyzed with a repeated measures analysis of variance (ANOVA) that included a within-subjects factor labelled as 'task' ('color task' versus 'shape task'). In addition to this factor, we used a within-subjects factor labelled as 'ROI' ('anterior' versus 'posterior') to compare the electrophysiological activity between anterior and posterior brain areas.

\section{Authors' contributions}

HJP has initiated this study. BKM has designed the experiment and drafted the manuscript. BKM and HJP carried out the recording of the data and ran the analyses and discussed the results. All authors have read and approved the final manuscript.

\section{Acknowledgements}

This research was supported by the Original Technology Research Program for Brain Science through the National Research Foundation of Korea (NRF) funded by the Ministry of Education, Science and Technology (MEST) (No. 20100018839) to HJP. We are thankful to Joong II Kim, Dong-Ha Lee, and Seong Yong Park for helping out during the acquisition of data.

\section{Author Details}

'Department of Radiology, Brigham and Women's Hospital, Harvard Medical School, Boston, USA and ${ }^{2}$ Brain Korea 21 Project for Medical Science, Department of Radiology, Nuclear Medicine and Research Institute of Radiological Science, Yonsei University College of Medicine, Seoul, Korea

Received: 9 December 2009 Accepted: 28 June 2010

Published: 28 June 2010

\section{References}

1. Brandt ME, Jansen BH: The relationship between prestimulus-alpha amplitude and visual evoked potential amplitude. Int J Neurosci 1991, 61(3-4):261-268.

2. Barry RJ, Kirkaikul S, Hodder D: EEG alpha activity and the ERP to target stimuli in an auditory oddball paradigm. Int J Psychophysiol 2000, 39(1):39-50.

3. Jansen $B H$, Brandt ME: The effect of the phase of prestimulus alpha activity on the averaged visual evoked response. Electroencephalogr Clin Neurophysiol 1991, 80(4):241-250.

4. Brandt ME, Jansen BH, Carbonari JP: Pre-stimulus spectral EEG patterns and the visual evoked response. Electroencephalogr Clin Neurophysiol 1991, 80(1):16-20

5. Brandt ME: Visual and auditory evoked phase resetting of the alpha EEG. Int J Psychophysiol 1997, 26(1-3):285-298.
6. Başar E, Stampfer HG: Important associations among EEG-dynamics, event-related potentials, short-term memory and learning. Int J Neurosci 1985, 26(3-4):161-180.

7. Rahn E, Başar E: Enhancement of visual evoked potentials by stimulation during low prestimulus EEG stages. Int J Neurosci 1993, 72(1-2):123-136.

8. Rahn E, Başar E: Prestimulus EEG-activity strongly influences the auditory evoked vertex response: a new method for selective averaging. Int J Neurosci 1993, 69(1-4):207-220.

9. Başar E: EEG-Brain Dynamics: Relation Between EEG and Brain Evoked Potentials. New York: Elsevier; 1980:375-391.

10. Donchin E: Event-related brain potentials in the study of human information processing. In Evoked brain potentials and behaviour Edited by: Begleiter H. New York: Plenum Press; 1979:13-88.

11. Hillyard SA, Kutas M: Electrophysiology of cognitive processing. Annu Rev Psychol 1983, 34:33-61.

12. Picton TW, Hillyard SA: Endogenous event-related potentials. In Handbook of Electroencephalography and Clinical Neurophysiology Volume 3. Edited by: Picton TW. Amsterdam: Elsevier; 1988:361-426.

13. Klimesch W, Sauseng P, Gruber W: The functional relevance of phase reset: a comment to Risner et al. (2009): The visual evoked potential of surface alpha rhythm phase. Neuroimage 2009, 47(1):5-7.

14. Freunberger R, Holler Y, Griesmayr B, Gruber W, Sauseng P, Klimesch W: Functional similarities between the $\mathrm{P} 1$ component and alpha oscillations. Eur J Neurosci 2008, 27(9):2330-2340.

15. Klimesch W, Schack B, Schabus M, Doppelmayr M, Gruber W, Sauseng P: Phase-locked alpha and theta oscillations generate the P1-N1 complex and are related to memory performance. Brain Res Cogn Brain Res 2004, 19(3):302-316.

16. Gruber WR, Klimesch W, Sauseng P, Doppelmayr M: Alpha phase synchronization predicts $\mathrm{P} 1$ and $\mathrm{N} 1$ latency and amplitude size. Cereb Cortex 2005, 15(4):371-377.

17. Mazaheri A, Jensen O: Posterior alpha activity is not phase-reset by visual stimuli. Proc Natl Acad Sci USA 2006, 103(8):2948-2952.

18. HansImayr S, Klimesch W, Sauseng P, Gruber W, Doppelmayr M, Freunberger R, Pecherstorfer T, Birbaumer N: Alpha phase reset contributes to the generation of ERPs. Cereb Cortex 2007, 17(1):1-8.

19. Risner ML, Aura CJ, Black JE, Gawne TJ: The Visual Evoked Potential is independent of surface alpha rhythm phase. Neuroimage 2009, 45(2):463-469.

20. Min BK, Herrmann CS: Prestimulus EEG alpha activity reflects prestimulus top-down processing. Neurosci Lett 2007, 422(2):131-135.

21. Ergenoglu T, Demiralp T, Bayraktaroglu Z, Ergen M, Beydagi H, Uresin Y: Alpha rhythm of the EEG modulates visual detection performance in humans. Brain Res Cogn Brain Res 2004, 20(3):376-383.

22. Yordanova J, Kolev V, Heinrich H, Woerner W, Banaschewski T, Rothenberger A: Developmental event-related gamma oscillations: effects of auditory attention. Eur J Neurosci 2002, 16(11):2214-2224.

23. Klimesch W, Sauseng P, HansImayr S: EEG alpha oscillations: the inhibition-timing hypothesis. Brain Res Rev 2007, 53(1):63-88.

24. Cooper NR, Croft RJ, Dominey SJ, Burgess AP, Gruzelier JH: Paradox lost? Exploring the role of alpha oscillations during externally vs. internally directed attention and the implications for idling and inhibition hypotheses. Int J Psychophysiol 2003, 47(1):65-74.

25. Jensen O, Gelfand J, Kounios J, Lisman JE: Oscillations in the alpha band $(9-12 \mathrm{~Hz})$ increase with memory load during retention in a short-term memory task. Cereb Cortex 2002, 12(8):877-882.

26. Klimesch W: EEG alpha and theta oscillations reflect cognitive and memory performance: a review and analysis. Brain Res Brain Res Rev 1999, 29(2-3):169-195.

27. Schack B, Klimesch W: Frequency characteristics of evoked and oscillatory electroencephalic activity in a human memory scanning task. Neurosci Lett 2002, 331(2):107-110.

28. Herrmann CS, Senkowski D, Rottger S: Phase-locking and amplitude modulations of EEG alpha: Two measures reflect different cognitive processes in a working memory task. Exp Psychol 2004, 51(4):311-318.

29. Suffczynski P, Kalitzin S, Pfurtscheller G, Lopes FH da Silva: Computational model of thalamo-cortical networks: dynamical control of alpha rhythms in relation to focal attention. Int J Psychophysiol 2001 43(1):25-40

30. Busch NA, Herrmann CS: Object-load and feature-load modulate EEG in a short-term memory task. Neuroreport 2003, 14(13):1721-1724. 
31. Mordkoff JT, Yantis S: Dividing attention between color and shape: evidence of coactivation. Percept Psychophys 1993, 53(4):357-366.

32. Başar E, Rahn E, Demiralp T, Schürmann M: Spontaneous EEG theta activity controls frontal visual evoked potential amplitudes. Electroencephalogr Clin Neurophysiol 1998, 108(2):101-109.

33. Freunberger R, Klimesch W, Doppelmayr M, Holler Y: Visual P2 component is related to theta phase-locking. Neurosci Lett 2007, 426(3):181-186

34. Sauseng P, Klimesch W, Gruber WR, Birbaumer N: Cross-frequency phase synchronization: A brain mechanism of memory matching and attention. Neurolmage 2008, 40(1):308-317.

35. Sauseng P, Klimesch W, Schabus M, Doppelmayr M: Fronto-parietal EEG coherence in theta and upper alpha reflect central executive functions of working memory. Int J Psychophysiol 2005, 57(2):97-103.

36. Von Stein A, Sarnthein J: Different frequencies for different scales of cortical integration: From local gamma to long range alpha/theta synchronization. International Journal of Psychophysiology 2000, 38(3):301-313.

37. Angelakis E, Lubar JF, Stathopoulou S, Kounios J: Peak alpha frequency: an electroencephalographic measure of cognitive preparedness. Clin Neurophysiol 2004, 115(4):887-897.

38. Foxe JJ, Simpson GV, Ahlfors SP: Parieto-occipital approximately $10 \mathrm{~Hz}$ activity reflects anticipatory state of visual attention mechanisms. Neuroreport 1998, 9(17):3929-3933.

39. Pfurtscheller G: Event-related synchronization (ERS): an electrophysiological correlate of cortical areas at rest. Electroencephalogr Clin Neurophysiol 1992, 83(1):62-69.

40. Min BK, Park JY, Kim EJ, Kim JI, Kim JJ, Park HJ: Prestimulus EEG alpha activity reflects temporal expectancy. Neurosci Lett 2008, 438(3):270-274

41. Pizzagalli DA, Oakes TR, Davidson RJ: Coupling of theta activity and glucose metabolism in the human rostral anterior cingulate cortex: An EEG/PET study of normal and depressed subjects. Psychophysiology 2003, 40(6):939-949.

42. Asada H, Fukuda Y, Tsunoda S, Yamaguchi M, Tonoike M: Frontal midline theta rhythms reflect alternative activation of prefrontal cortex and anterior cingulate cortex in humans. Neuroscience Letters 1999, 274(1):29-32

43. Wang C, Ulbert I, Schomer DL, Marinkovic K, Halgren E: Responses of human anterior cingulate cortex microdomains to error detection, conflict monitoring stimulus-response mapping, familiarity, and orienting. Journal of Neuroscience 2005, 25(3):604-613.

44. Yordanova J, Falkenstein M, Hohnsbein J, Kolev V: Parallel systems of error processing in the brain. Neuroimage 2004, 22(2):590-602.

45. Carter CS, Braver TS, Barch DM, Botvinick MM, Noll D, Cohen JD: Anterior cingulate cortex error detection and the online monitoring of performance. Science 1998, 280(5364):747-749.

46. HansImayr S, Pastötter B, Bäuml KH, Gruber S, Wimber M, Klimesch W: The electrophysiological dynamics of interference during the stroop task. Journal of Cognitive Neuroscience 2008, 20(2):215-225.

47. Scheeringa R, Petersson KM, Oostenveld R, Norris DG, Hagoort P, Bastiaansen MC: Trial-by-trial coupling between EEG and BOLD identifies networks related to alpha and theta EEG power increases during working memory maintenance. Neuroimage 2009, 44(3):1224-1238.

48. Nunez PL, Wingeier BM, Silberstein RB: Spatial-temporal structures of human alpha rhythms: theory, microcurrent sources, multiscale measurements, and global binding of local networks. Hum Brain Mapp 2001, 13(3):125-164.

49. Sauseng P, Klimesch W, Gruber W, Doppelmayr M, Stadler W, Schabus M: The interplay between theta and alpha oscillations in the human electroencephalogram reflects the transfer of information between memory systems. Neuroscience Letters 2002, 324(2):121-124.

50. Babiloni C, Ferri R, Moretti DV, Strambi A, Binetti G, Dal Forno G, Ferreri F, Lanuzza B, Bonato C, Nobili F, et al:: Abnormal fronto-parietal coupling of brain rhythms in mild Alzheimer's disease: A multicentric EEG study. European Journal of Neuroscience 2004, 19(9):2583-2590.

51. Sauseng P, Klimesch W, Doppelmayr M, Pecherstorfer T, Freunberger $R$, HansImayr S: EEG alpha synchronization and functional coupling during top-down processing in a working memory task. Human Brain Mapping 2005, 26(2):148-155.
52. Herrmann CS, Grigutsch M, Busch NA: EEG oscillations and wavelet analysis. In Event-related potentials: a methods handbook Edited by: Handy TC. Cambridge: The MIT Press; 2005:229-259.

53. Herrmann CS, Mecklinger A: Magnetoencephalographic responses to illusory figures: early evoked gamma is affected by processing of stimulus features. Int J Psychophysiol 2000, 38(3):265-281.

54. Yordanova J, Heinrich H, Kolev V, Rothenberger A: Increased eventrelated theta activity as a psychophysiological marker of comorbidity in children with tics and attention-deficit/hyperactivity disorders. Neuroimage 2006, 32(2):940-955

55. Yordanova J, Kolev V: Single-sweep analysis of the theta frequency band during an auditory oddball task. Psychophysiology 1998, 35(1):116-126.

doi: 10.1186/1471-2202-11-79

Cite this article as: Min and Park, Task-related modulation of anterior theta and posterior alpha EEG reflects top-down preparation BMC Neuroscience 2010, 11:79

\section{Submit your next manuscript to BioMed Central and take full advantage of:}

- Convenient online submission

- Thorough peer review

- No space constraints or color figure charges

- Immediate publication on acceptance

- Inclusion in PubMed, CAS, Scopus and Google Scholar

- Research which is freely available for redistribution

Submit your manuscript at www.biomedcentral.com/submit
C) Biomed Central 